\title{
Autoimmune Haemolytic Anaemia Following Influenza A Pandemic H1N1 Infection: A Case Report
}

\section{Pritam Bandyopadhyay, Tamoghna Biswas and Mahuya Lahiri}

Department of Paediatrics, ESI-PGIMSR, ESIC Medical College and Hospital, Kolkata, India

Correspondence:
Pritam Bandyopadhyay
Department of Paediatrics
ESI-PGIMSR,
ESIC Medical College and Hospital,
Kolkata. India
Email: pritambanerjee86@gmail.com

DOI: $10.3126 /$ jnps.v39i2.27878

Submitted on: 2020-03-01

Accepted on: 2020-04-10

Acknowledgements: None

Funding: Nil

Conflict of Interest: None declared

Permission form IRB: Yes

To cite this article: Bandyopadhyay $\mathrm{P}$, Biswas T, Lahiri M. Autoimmune hemolytic anemia following influenza a pandemic H1N1 infection: a case report. J Nepal Paediatr Soc. 2019;39(2):116-8.

\section{ABSTRACT}

Autoimmune haemolytic anaemia has been rarely reported in association with influenza virus. Here, we report a 16 months old girl with warm autoimmune haemolytic anaemia following influenza A Pandemic H1N1 infection, which improved with oral corticosteroids and intravenous immunoglobulin.

Key words: AIHA (autoimmune haemolytic anemia); Influenza A (H1N1) infection; IVIG (Intravenous Immunoglobulin) administration 


\section{INTRODUCTION}

Influenza $\mathrm{A} /(\mathrm{H} 1 \mathrm{~N} 1)$ pdm09 virus is a significant cause of mortality and morbidity across the globe, even in the post-pandemic period. While majority of the infected population recovers uneventfully, the severity is more pronounced in vulnerable age groups like children who are prone to suffer from complications of the disease. Here, we present a case of influenza A/(H1N1) pdm09 with autoimmune haemolytic anaemia, a rarely reported association.

\section{CASE REPORT}

A 16 months old girl from South 24 Pargana district of West Bengal, India was admitted in our paediatric intensive care unit with a history of cough and coryza for five days followed by high grade fever and respiratory distress with inability to suck well for one day. On admission, the child was lethargic, and tachypneic with marked intercostal and subcostal retractions with oxygen saturation below $90 \%$ in room air. Auscultation revealed bilateral wheeze and crackles with decreased air entry on the right side. Initial chest $\mathrm{X}$ ray was suggestive of right sided pneumonia. With the provisional diagnosis of right upper lobar pneumonia, child was started on empirical antibiotics with intravenous amoxicillinclavulunate which was subsequently upgraded to intravenous meropenem and vancomycin due to the deteriorating respiratory status. Supportive treatment was continued which included supplemental oxygen, intravenous fluids and nebulisation with salbutamol and ipratropium. Blood and urine cultures were negative. Nucleic Acid Amplification test done on throat swab specimen at the regional virus diagnostic laboratory, was positive for Influenza A Pandemic H1N1 infection. The child was started on a course of antiviral oral oseltamivir $30 \mathrm{mg} \mathrm{BD}$ for five days. The child showed dramatic improvement within 48 hours of starting oseltamivir with complete subsidence of respiratory symptoms within five days. However, after 10 days of admission, a low grade fever persisted and the child was developing clinically significant pallor, which was absent at the time of admission. Complete haemogram showed anaemia (Haemoglobin $5.1 \mathrm{~g} /$
$\mathrm{dL}$, red cell count $\left(1.1 \mathrm{X} 10^{6} / \mu \mathrm{L}\right)$ with leucocytosis (Total leucocyte count $22700 / \mu \mathrm{L}$ with $59 \%$ neutrophils, $32 \%$ lymphocytes, $6 \%$ monocytes and $3 \%$ eosinophils). Reticulocytosis (reticulocyte count $3.5 \%$ ) and elevated lactate dehydrogenase (1340 U/L) suggested an acquired haemolytic anaemia. An immune mediated process was suspected as there was problem in cross matching for blood group determination as reported in our blood bank. Polyspecific direct antiglobulin test (DAT) or direct Coombs test was positive, and monospecific Coombs test showed IgG positivity at 37 degree celsius typical of warm autoimmune haemolytic anaemia. Intravenous immunogloubulin (IVIG) was administered and oral prednisolone was started at a dose of $2 \mathrm{mg} / \mathrm{kg} /$ day. An increase in haemoglobin was apparent within five days after start of steroids, and the child was discharged in a clinically stable condition with tapering course of oral corticosteroids. On follow up after two weeks, DAT was negative and haemoglobin level was normal without blood transfusion.

\section{DISCUSSION}

AIHA is a relatively uncommon disease in children. While accurate estimates of incidence are lacking, a French observational study reported a rate of 0.2 cases $/ 1,000,000$ in under-20 population. ${ }^{1}$ AIHA is characterised by premature lysis of red blood corpuscles mediated by autoantibodies, depending on the thermal range of which the disease is classified into warm antibody, cold antibody and mixed type AIHA. Secondary AIHAs have been reported to be associated with infections, drug exposure, autoimmune disorders, lymphoproliferative disorders, malignancies etc. Among infections causing secondary AIHA, Mycoplasma pneumonia, Epstein-Barr virus, cytomegalovirus, parvovirus, hepatitis $\mathrm{A}$ and $\mathrm{E}$ virus, etc are significant. However, influenza virus has been rarely reported to be associated with AIHA. ${ }^{2}$ Till date, only a few case reports exist in the global scientific literature about a possible association between influenza virus infection and secondary AIHA. ${ }^{3-6}$ In 2009, Schoindre et al. reported a case of cold agglutinin syndrome (CAS) in a 60 years old woman with diabetic nephropathy following influenza A H1N1 infection. ${ }^{3}$ Cold 
agglutinin disease following influenza A infection has also been reported by Ahn et al. in a 58 years old female from Brazil. ${ }^{4}$ Shizuma et al. reported a case of AIHA following influenza $\mathrm{A}$ in a patient with alcoholic liver disease, who responded initially to prednisolone, but later succumbed due to hepatorenal syndrome. ${ }^{5}$ Recently in 2017, a paediatric center from North India has also reported a case of AIHA in a one year old girl with influenza A H1N1 infection, who responded favourably to treatment with oseltamivir and intravenous immunoglobulin. ${ }^{6}$ Our patient was treated with both oral steroids and IVIG, in view of the fulminant clinical course and considering the increased likelihood of severe complications in this vulnerable age group. Although our patient received Oseltamivir, it is an antiviral agent against Influenza A (H1N1) infection, but the medicine is not a known agent to cause drug induced AIHA. ${ }^{8}$ However, there has been a report where Oseltamivir led to AIHA, but there also AIHA was recovered two weeks after withdrawal of antiviral agent and concomitant steroid therapy. ${ }^{9}$ Generally, in drug induced cases, response is immediate following omission of the drug. So here in our case, AIHA is more likely to have been caused by H1N1 infection itself, rather than oseltamivir induced. A case reported by Chen $\mathrm{H} 1$ et al. that AIHA with thrombocytopenia treated with Methylprednisolone had succumbed due to multiorgan failure. ${ }^{7}$

\section{CONCLUSIONS}

This case presentation suggested that though rare, autoimmune haemolytic anaemia can be a potentially devastating complication of influenza A infection. Keeping a low threshold of suspicion is imperative for early recognition and prompt treatment of this condition.

\section{REFERENCES}

1. Aladjidi N, Leverger G, Leblanc T, Picat MQ, Michel G, Bertrand Y, et al. Centre de Référence National des Cytopénies Auto-immunes de l'Enfant (CEREVANCE). New insights into childhood autoimmune hemolytic anemia: a French national observational study of 265 children. Haematologica. 2011 May;96(5):655-63. DOI: 10.3324/ hematol.2010.036053.

2. Shizuma T. Autoimmune hemolytic anemia following influenza virus infection or administration of influenza vaccine. J Blood Disorders Transf. 2014;5(3):1000200. DOI: 10.4172/2155-9864.1000200

3. Schoindre Y, Bollée G, Dumont MD, Lesavre P, Servais A. Cold agglutinin syndrome associated with a 2009 influenza A H1N1 infection. Am J Med. 2011 Feb;124(2):e1-2.DOI: https://doi.org/10.1016/j.amjmed.2010.05.015

4. Ahn M, Rehr MJ, Santarelli GE. Cold hemolytic anemia: a rare complication of influenza A. JCSO. 2017;15(6): 335-8. DOI :https://doi.org/10.12788/jcso.0346

5. Shizuma T. A case of autoimmune hemolytic anemia caused by type A influenza infection in a patient with alcoholic liver cirrhosis. Kansenshogaku Zasshi. 2010 May;84(3):296-9. DOI: 10.11150/kansenshogakuzasshi.84.296

6. Singh A, Mandal A, Patel A, Mishra S. Autoimmune Haemolytic Anaemia-A Spectrum of Presentation in Children. J Clin Diagn Res. 2017 Sep;11(9):SR01-SR02. DOI: 10.7860/JCDR/2017/27895.10646

7. Chen H1, Jia XL, Gao HM, Qian S. Comorbid presentation of severe novel influenza (H1N1) and Evans syndrome: a case report. Chin Med J (Clin Res Ed). 2011;282:1743-6. DOI: 10.3760/cma.j.issn.0366- 69999.2011.11.026

8. Garratty G. Immune hemolytic anemia associated with drug therapy. Blood Rev. 2010;24:143-50. DOI: https:// doi.org/10.1016/j.blre.2010.06.004

9. Yagi Y, Yagi Y, Sageshima H, Miyata Y. A case of infant with hemolytic anemia after oral oseltamivir administration. Acta Paediatr Jpn. 2004;108: 649 - 651. DOI: https://ci.nii.ac.ip/naid/10014204484\#cinii 